ISSN: 2591-7994

\title{
Comparison of follicular fluid concentrations of anti-mullerian hormone, bone morphogenetic protein 15 and transforming growth factor $\$ 1$ between modified natural and stimulated in-vitro fertilization cycles.
}

\author{
Tanja Burnik Papler, Eda Vrtačnik Bokal, Rok Devjak, Nina Jančar* \\ Department of Human Reproduction, Division of Obstetrics and Gynecology, University Medical Centre Ljubljana, \\ Ljubljana, Slovenia
}

\begin{abstract}
Analysis of biochemical characteristics of follicular fluid (FF) can serve as a non-invasive method for the prediction of oocyte and embryo quality. The aim of the present study was to compare concentrations of anti-mullerian hormone, bone morphogenetic protein 15 and transforming growth factor $\mathrm{B1}$ in $\mathrm{FF}$ of follicles giving rise to high quality embryos between natural and stimulated IVF cycles. Concentration of BMP-15 was significantly higher and concentration of TGF-ß1 was significantly lower in stimulated IVF cycles. Concentrations of markers of oocyte and embryo quality were significantly different between natural and stimulated IVF cycles. This could be the cause for lower pregnancy rates in natural IVF cycles.
\end{abstract}

Keywords: Modified natural cycle, Stimulated cycle, Follicular fluid, Anti-mullerian hormone, Bone morphogenetic protein 15, Transforming growth factor $\beta 1$.

Accepted on July 25, 2018

\section{Introduction}

There has been a recent tendency towards modified natural IVF (MNIVF) cycles to avoid the most common complications of controlled ovarian hyperstimulation $(\mathrm{COH})$ cycles, such as multiple pregnancies and Ovarian hyperstimulation syndrome. However, MNIVF is associated with low pregnancy rate.

Follicular fluid (FF) is the microenvironment of oocyte development and maturation; therefore its composition could reflect oocyte quality. It has been proposed that concentrations of certain FF proteins correlate with oocyte and embryo quality [1].

The aim of the present study was to compare concentrations of anti-mullerian hormone (AMH), bone morphogenetic protein 15 (BMP-15) and transforming growth factor $\beta 1$ (TGF- 31 ) in FF between MNIVF and $\mathrm{COH}$ cycles.

\section{Materials and Methods}

Forty-three women with primary infertility aged $<35$ years with tubal or unexplained infertility with normal partners spermiogram were included in the study; 20 undergoing their first cycle of $\mathrm{COH}$ and 23 undergoing their first MNIVF. In the $\mathrm{COH}$ group, recombinant FSH folitropin beta was injected subcutaneously (Puregon; Organon, Dublin, Ireland) from day 2 of the cycle with the addition of GnRH antagonist (Cetrotide; Merck Serono, Darmstadt, Germany) after dominant follicle measured $\geq 13 \mathrm{~mm}$. For final oocyte maturation, 10,000 IU of human chorionic gonadotropin (hCG) (Pregnyl; N.V Organon, Oss, the Netherlands) were administered, and oocyte retrieval was carried out 34 to 36 hours later. In the MNIVF group only
hCG for triggering of final oocyte maturation was used to modify the natural cycle. Transvaginal ultrasonographic examination and erum E2 measurement was performed daily from day 9 of the menstrual cycle. When the dominant follicle measured $\geq 16 \mathrm{~mm}$ in diameter, serum E2 exceeded $0.39 \mathrm{nmol} / 1$, and no LH surge was detected in the urine sample, 5,000 IU of hCG (Pregnyl; N.V. Organon, Oss, The Netherlands) were injected subcutaneously. Oocyte retrieval was carried out after 31 to 32 hours. The study was approved by the National Medical Ethics Committee and all patients signed informed consent.

Each follicle was aspirated individually. Oocytes were fertilized using ICSI and embryos were individually cultured for 5 days. A total of 68 follicles whose embryos developed to morulae or blastocysts on day 5 were analyzed; 45 derived from $\mathrm{COH}$ and 23 from MNIVF cycles. FF were centrifuged at $2500 \mathrm{~g}$ for 10 minutes and stored at $-80^{\circ} \mathrm{C}$ until analysis. Enzymelinked immunosorbent assay (ELISA) was used for protein concentration measurements. Statistical analysis was performed with statistical package IBM SPSS Statistics 22.0 (IBM Corporation, Armonk, USA) using independent samples t-test.

\section{Results}

There were no significant differences in the mean patient's age, body mass index and serum concentration of $\mathrm{AMH}$ on the day of oocyte retrieval between the two groups. But there was a difference in serum concentration of FSH on the day of oocyte retrieval between the two groups, which is shown in Table 1. Concentrations of AMH, BMP-3B, BMP-15 and TGF- $\beta 1$ in FF of MNIVF and $\mathrm{COH}$ cycles with corresponding $P$ values are also presented in Table 1 . 
Citation: Papler TB, Bokal EV, Devjak R, et al. Comparison of follicular fluid concentrations of anti-mullerian hormone, bone morphogenetic protein 15 and transforming growth factor $\beta 1$ between modified natural and stimulated in vitro fertilization cycles. Gynecol Reproduct Endocrinol -UK. 2018;2(2):1-2

Table 1: Patients characteristics and protein concentrations.

\begin{tabular}{|c|c|c|c|}
\hline & MNIVF & $\mathrm{COH}$ & P value \\
\hline Mean age (years) & $34.9 \pm 3.4$ & $33.6 \pm 4.1$ & NS \\
\hline $\begin{array}{l}\text { Body mass index }(\mathrm{kg} / \\
\left.\mathrm{m}^{2}\right)\end{array}$ & $21.9 \pm 2.5$ & $23.6 \pm 4.3$ & NS \\
\hline Serum FSH (IU/L) & $12.9 \pm 5.9$ & $6.3 \pm 2.6$ & $<0.001$ \\
\hline Serum AMH (ng/mL) & 2.32 .0 & $1.4 \pm 0.9$ & NS \\
\hline Follicular AMH (ng/L) & $\begin{array}{c}731.24 \pm \\
347.76\end{array}$ & $\begin{array}{c}646.69 \pm \\
267.41\end{array}$ & NS \\
\hline $\begin{array}{c}\text { Follicular BMP-15 } \\
(\mathrm{ng} / \mathrm{L})\end{array}$ & $50.67 \pm 56.05$ & $\begin{array}{c}165.27 \pm \\
214.75\end{array}$ & 0.02 \\
\hline Follicular TGF-ß1 (ng/L) & $\begin{array}{c}1925.10 \pm \\
724.74\end{array}$ & $\begin{array}{c}1546.12 \pm \\
459.73\end{array}$ & 0.04 \\
\hline $\begin{array}{c}\text { Pregnancy rate per cycle } \\
(\%)\end{array}$ & 13.04 & 35.0 & \\
\hline
\end{tabular}

MNIVF-modified natural IVF; COH-controlled ovarian hyperstimulation; FSH-follicle stimulating hormone; AMH-antimullerian hormone; BMP-15- bone morphogenetic protein 15; TGF-ß1- transforming growth factor $ß 1$.

Concentration of BMP-15 was significantly higher in FF giving rise to high quality embryos in $\mathrm{COH}$ when compared to MNIVF cycles. Concentration of TGF- 11 was significantly higher in FF of MNIVF when compared to $\mathrm{COH}$ cycles. Concentration of AMH in FF was similar in both groups. Pregnancy rate per cycle in the two groups is also shown in Table 1.

\section{Discussion}

The results of our study have shown that there are differences in protein concentrations of FF in MNIVF and $\mathrm{COH}$ cycles even between embryos of morphologically similar quality. The analysis was deliberately performed only in FF of high quality embryos-morulae and blastocysts, which are the two developmental stages on day 5 of in vitro culture, capable of implantation and resulting in clinical pregnancy. We assumed that oocytes, which can develop to embryos of morphologically similar quality on day 5 of IVF cycle, should also have similar concentrations of proteins related to embryo quality in FF. However, concentration of BMP-15 was higher and TGF- 31 was lower in $\mathrm{FF}$ of $\mathrm{COH}$ cycles when compared to MNIVF cycles.

The BMP-15 concentration in FF was proposed as a potential biomarker of oocyte quality. Higher FF concentration of BMP15 was associated with better oocyte and embryo quality and correlated with higher implantation, pregnancy and live birth rates [2]. The lower concentration of BMP-15 in MNIVF cycles might thus be associated with lower pregnancy rates when compared to $\mathrm{COH}$ cycles.

According to the data of a previous study by Zheng TGF- 11 inhibits luteinization and induces apoptosis of bovine granulosa cells (GC) [3]. Continued survival of GC following ovulation and their luteinization was critical for progesterone production and the ability to sustain pregnancy, while higher incidence of apoptotic GC was associated with lower implantation rate as documented in our previous study [4]. Based on the connection between TGF- $\beta 1$ and apoptosis, lower concentration of TGF- $\beta 1$ in $\mathrm{FF}$ of $\mathrm{COH}$ cycles could predict better pregnancy rates in these cycles.

Concentration of $\mathrm{AMH}$ in FF was found to be associated with embryo quality [5]. No difference was found in FF concentrations of AMH between $\mathrm{COH}$ and MNIVF cycles in the present study. Similarity of FF AMH concentrations between both groups might be due to selective inclusion of FF from follicles with ensuing high quality embryos and resulting in clinical pregnancy.

Based on the results of our study, we can conclude that higher pregnancy rates in $\mathrm{COH}$ cycles can also be explained by different concentrations of certain FF proteins when compared to MNIVF cycles. Higher concentration of BMP-15 and lower concentration of TGF- $\beta 1$ in $\mathrm{FF}$ with ensuing high quality embryos might both be associated with higher pregnancy rates in $\mathrm{COH}$ cycles.

\section{References}

1. Revelli A, Delle Piane L, Casano S, et al. Follicular fluid content and oocyte quality: From single biochemical markers to metabolomics. Reprod Biol Endocrinol 2009; $7: 40-52$.

2. Wu YT, Wang TT, Chen XJ, et al. Bone morphogenetic protein-15 in follicle fluid combined with age may differentiate between successful and unsuccessful poor ovarian responders. Reprod Biol Endocrinol 2012;10:11621.

3. Zheng X, Boerboom D, Carrière PD. Transforming growth factor-beta1 inhibits luteinization and promotes apoptosis in bovine granulosa cells. Reproduction 2009;137:969-77.

4. Jancar N, Virant-Klun I, Osredkar J, et al. Apoptosis reactive oxygen species and follicular anti-müllerian hormone in natural versus stimulated cycles. Reprod Biomed Online 2008;5:640-8.

5. Aflatoonian A, Mashayekhy M, Mohamadian F, et al. The correlation between follicular fluid anti-mullerian hormone levels and fertilization and embryo quality in ART cycles. Iran J Reprod Med 2010;8:157-60.

\section{*Correspondence to:}

Nina Jancar

Department of Human Reproduction

University Medical Centre Ljubljana

Slovenia

Tel: 38-615-226295

E-mail: nina.jancar@kclj.si 